\section{MS34-P2 Mixed Metal Multinuclear Cr(III) Cage Compounds and Coordination Polymers Based on Unsubstituted
Phenolate: Design, Synthesis, Mechanism, and Properties.

Aurelien Crochet ${ }^{1}$, Jean-Pierre Brog ${ }^{1}$, Katharina M. Fromm ${ }^{1}$

\section{Department of Chemistry, University of Fribourg, Switzerland}

email: aurelien.crochet@unifr.ch

The use of alkali aryloxide reagents in organo-metallic synthesis often depends on their solubility, a property derived from their structure. The regain of interest of alkali aryloxides also originates from the discovery of high-temperature superconducting compounds, which has generated a great interest in the formation of oxide materials and other ceramics. Thus, many alkoxides of yttrium and copper are common precursors for oxide materials. Moreover, the synthesis of heterobimetallic alkoxides has provided a facile route for obtaining soluble, volatile, and generally monomeric species. These heterobimetallic complexes can thus serve as valuable precursors for making mixed metal oxides. This is however not the only possible application for this type of compounds. These complexes can be used as starting compounds for the syntheses of more complex structures. In this work, the salt elimination and ligand exchange reaction of chromium(II) chloride with lithium phenoxide yields a mixed metal lithium-chromium(II) phenoxide. Using this latter as intermediately formed starting material and combining the substitution reaction with an oxidation process, we have gained access to new polynuclear chromium(III) aryloxide complexes. While a $1 \mathrm{D}$ coordination polymer based on chromium(III) is obtained in a first reaction by serendipity, the controlled addition of water to the Cr(II) complex leads to three new discrete chromium(III) cluster compounds. The use of deuterated species allowed to confirm the oxidation based on the addition of water by detection of H2, HD and D2. During these investigations, we have also identified a THF-adduct of chromium(II) chloride, used in the literature as precursor in numerous syntheses, but with a hitherto unknown structure.

A. Crochet, J-P. Brog, and K. M. Fromm, Cryst. Growth Des., 2016, 16 (1), pp 189-199.

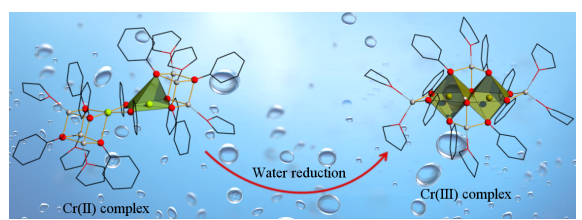

Figure 1. Oxidation of a $\mathrm{Cr}$ (II) complex by water and formation of a new $\mathrm{Cr}$ (III) complex and hydrogen.

Keywords: Chromium, oxidation, reduction, aryloxide, water

\section{MS34-P3 Fabrication of New MOFs via Rational Design of the Organic Building Block}

Rajdip Dey ${ }^{1}$, Susan A Bourne ${ }^{1}$

1. Centre for Supramolecular Chemistry Research, Department of Chemistry, University of Capetown,7700 Rondebosch, South Africa

email: rajdipdey@gmail.com

The fabrication of metal organic frameworks ${ }^{1}$ (MOFs) is one of the current interest in the fields of supramolecular chemistry and crystal engineering, not only because of their intriguing architectures but also due to its multidirectional functional property. ${ }^{2}$ Thus the synthesis and characterization of infinite one, two, and three dimensional (1D, 2D, and 3D) networks, with coordination space, has been an area of rapid growth. The applications of $\mathrm{MOFs}^{3}$ can be enhanced by the incorporation of open metal sites into the framework. As a result, development of methodologies for the inclusion of various metal centres into MOFs are important. It has been found that the metal component in MOFs play a critical role in the material's property. ${ }^{4}$ We have designed an important synthetic strategy and the use of metalloligand building blocks to incorporate various metal centres into MOFs.

Here we have synthesised several MOFs by the solvo-thermal process in a reaction with different metal system (Cu(II), Ni(II), Mn(II),Co(II), $\mathrm{Cd}(\mathrm{II}), \mathrm{Zn}(\mathrm{II})$ and using different dicarboxylates $\left(4,4^{\prime}, 4^{\prime \prime}, 4^{\prime \prime \prime}\right.$-benzene-1,2,4,5-tetrayltetrabenzoic acid, terphenyl- 3,3",5,5"-tetracarboxylic acid, 2,6-naphthalenedicarboxylic acid) and some newly designed $\mathrm{N}, \mathrm{N}$ donor ligands. The compounds have been analysed by PXRD, TGA and the single crystal X-ray structure. The propensity of these compounds to absorb solvent \& gas molecules commensurate with their porous structures are under study.

\section{References:}

1. O. M. Yaghi, M. O'Keeffe, N. W. Ockwig, H. K. Chae, M. Eddaoudi and J. Kim, Nature 2003, 423, 705.

2. S. Kitagawa, R. Kitaura and S. Noro, Angew. Chem., Int. Ed. 2004, 43, 2334.

3. J. Hennessy, Nature Materials, 2015, 14, 962.

4. R. Dey, R. Haldar, T. K. Maji and D.Ghoshal Cryst. Growth \& Des., 2011, 11, 3905.

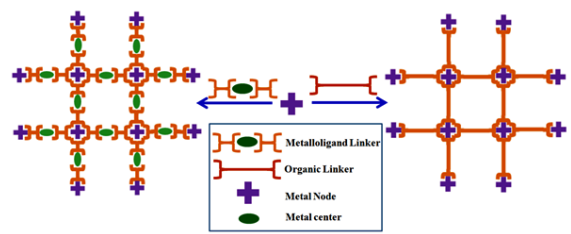

Figure 1. Scheme 1: The assembly of framework with an organic ligand linker versus a metalloligand linker allowing for the incorporation of various metal centers into the MOF

Keywords: MOF, dicarboxylate, Supramolecular Chemistry, Crystal engineering 